\title{
Cobalt-Catalyzed Enantioselective Hydrogenation of Enamides
}

Previous work on cobalt catalysts relied on activation by alkyl lithium reagents:

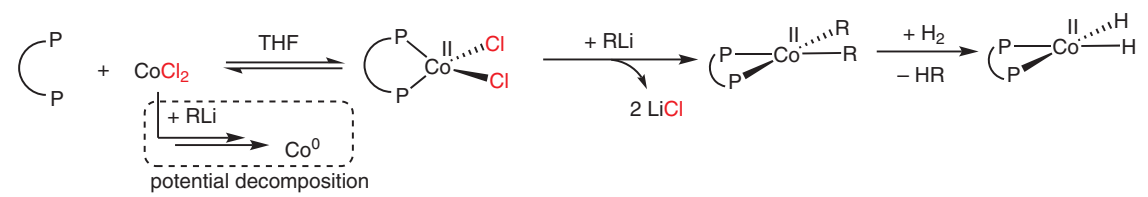

\section{enamides}

single-electron

reduction

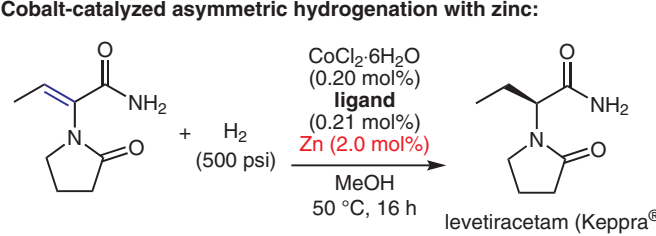

Mechanism:

dehydro-levetiracetam
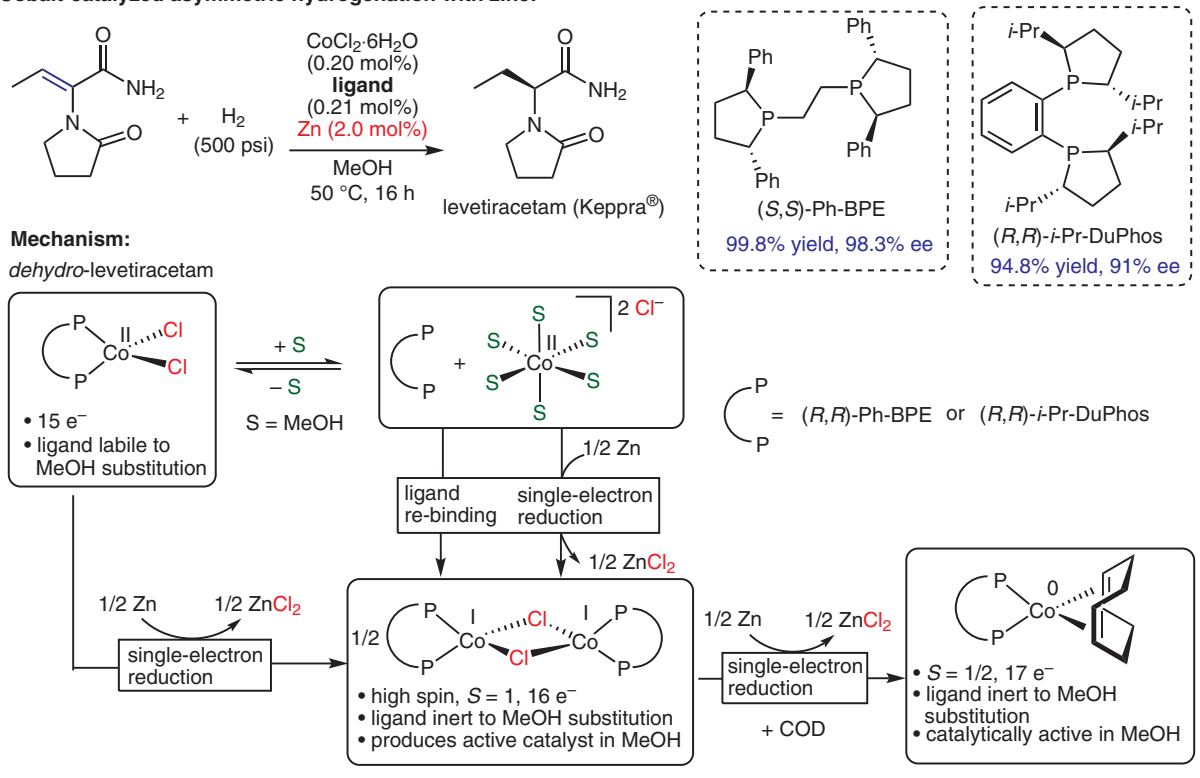

$94.8 \%$ yield, $91 \%$ ee

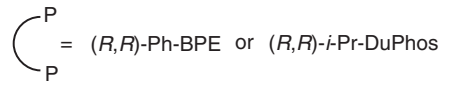

Zn-free hydrogenation:
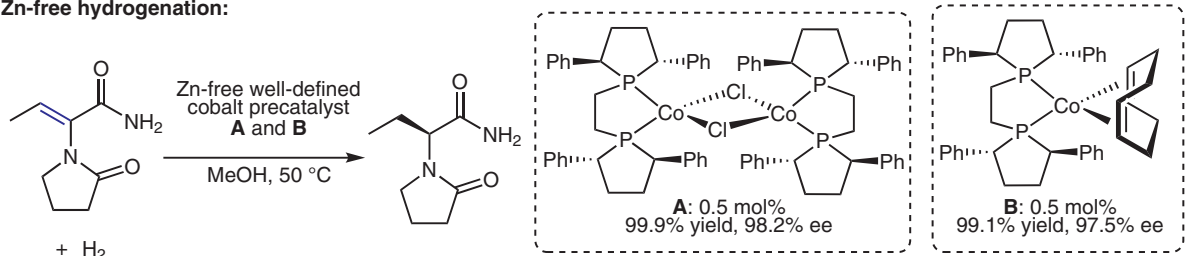

Significance: On the basis of a discovery from high-throughput reaction studies, the authors have developed a low-catalyst-loading enantioselective hydrogenation of functionalized alkenes through activation by zinc instead of the moreusual alkyl reagents. The optimized catalytic system $\mathrm{CoCl}_{2} \cdot 6 \mathrm{H}_{2} \mathrm{O} /(R, R)$-Ph-BPE or $\mathrm{CoCl}_{2} \cdot 6 \mathrm{H}_{2} \mathrm{O} /$ $(R, R)-i$-Pr-DuPhos realized a high reactivity and enantioselective hydrogenation in $\mathrm{MeOH}$.
Comment: A series of mechanistic studies revealed that $\mathrm{Co}(\mathrm{II})$ metal dissociates from the $\mathrm{Co}(\mathrm{II}) /$ phosphine complex in $\mathrm{MeOH}$, and that $\mathrm{Zn}$ reduces $\mathrm{Co}$ (II) to $\mathrm{Co}(\mathrm{I})$ through one-electron reduction to form a more stable Co-phosphine bond, which is key to the efficient enantioselective reduction of alkenes. The hydrogenation can be applied to a large-scale reaction requiring only $0.08 \mathrm{~mol} \%$ of the cobalt catalyst.

SYNFACTS Contributors: Hisashi Yamamoto, Takahiro Sawano Synfacts 2018, 14(08), 0819 Published online: 18.07.2018 DOI: 10.1055/s-0037-1610460; Reg-No.: H07818SF 